\title{
Outcomes and Prognostic Factors in Pediatric Oligodendroglioma: A Population-Based Study
}

\author{
Nicholas J. Goel ${ }^{\mathrm{a}}$ Kalil G. Abdullah ${ }^{\mathrm{b}}$ Shih-Shan Lang ${ }^{\mathrm{b}, \mathrm{c}}$ \\ a Perelman School of Medicine and ${ }^{b}$ Department of Neurosurgery, University of Pennsylvania, and ${ }^{\mathrm{C}}$ Division of \\ Neurosurgery, The Children's Hospital of Philadelphia, Philadelphia, PA, USA
}

\section{Keywords}

Surveillance, Epidemiology, and End Results database .

Pediatric oligodendroglioma · Pediatric cancer

\begin{abstract}
Background/Aims: Pediatric oligodendroglioma (pODG) is a rare primary brain tumor that remains poorly understood. Demographics, outcomes, and prognostic factors were analyzed in 346 pODG cases from the Surveillance, Epidemiology, and End Results database. Methods: Gender, race, age, tumor location, tumor size, tumor grade, extent of resection, and use of radiotherapy were evaluated with respect to overall survival (OS) by univariate and multivariate analysis. These factors were assessed in the pediatric cohort and 5,753 adult oligodendroglioma cases for comparison. Results: The mean OS in PODG was 199.6 months. Five- and 10-year survival rates were 85 and $81 \%$. pODG arose less frequently in the frontal lobe than adult tumors (53 vs. $22 \%$ ) but was more common in the temporal lobe (32 vs. 18\%) and extracortical regions ( 19 vs. $5 \%, p<0.0001$ ). pODG presented with smaller size (55 vs. $24 \%, p<0.0001$ ) and lower grade (72 vs. $54 \%, p<$ $0.0001)$ than adult tumors. Tumor location, size, grade, use of radiotherapy, and extent of resection were significant prognostic factors. Size and grade were much stronger prog-
\end{abstract}

\section{KARGER}

(C) 2017 S. Karger AG, Basel nostic factors in children than adults. While children with oligodendroglioma survive much longer than adults on the whole, there was no difference in outcome between children with high-grade tumors and adults with high-grade tumors. Conclusion: pODG differs significantly from adult oligodendroglioma along a number of demographic and tumor factors at a population level, and key prognostic factors influence survival differently in pODG than in adult disease.

(c) 2017 S. Karger AG, Basel

\section{Introduction}

Pediatric oligodendroglioma ( $\mathrm{pODG}$ ) remains a poorly understood entity, due in large part to its rarity. While primary brain tumors are now the leading cause of cancer-related death in children, only $1-5 \%$ of these tumors are classified as oligodendroglioma [1-3]. The standard of care for pODG includes maximal safe surgical resection, radiation, and a regimen of chemotherapy that differs across institutions and clinical trials. Because of its locally aggressive nature, oligodendroglioma is rarely cured by standard therapy and, despite a lengthy clinical course, is almost always fatal [4]. The median overall survival among all age groups is $10-14$ years [4-6].

Nicholas J. Goel

Perelman School of Medicine, University of Pennsylvania

3400 Civic Center Blvd.

Philadelphia, PA 19104 (USA)

E-Mail Nicholas.Goel@uphs.upenn.edu 
In recent years, genetic biomarkers have become essential to the diagnosis and management of oligodendroglioma. The previous $2007 \mathrm{WHO}$ Classification of Tumors of the Central Nervous System [7] listed diagnoses of "oligodendroglioma" and "anaplastic oligodendroglioma," which were based exclusively on histological phenotype. Among tumors classified by these guidelines, the $1 \mathrm{p} / 19 \mathrm{q}$ chromosomal codeletion was found in $40-80 \%$ of tumors $[8,9]$, and mutation in isocitrate dehydrogenase 1 (IDH1) was observed in $85-95 \%$ of tumors [10]. Both of these genetic markers are associated with a better response to standard therapy and better outcome $[11,12]$. In the recently released 2016 WHO classification, the presence of these markers has become essential to the diagnosis of an oligodendroglial tumor [13]. Primary diagnostic categories are now "oligodendroglioma, IDHmutant and $1 \mathrm{p} / 19 \mathrm{q}$ codeleted" and "anaplastic oligodendroglioma, IDH-mutant and $1 \mathrm{p} / 19 \mathrm{q}$ codeleted." The classifications of "oligodendroglioma, NOS" and "anaplastic oligodendroglioma, NOS" may be used in the case that genetic testing is not available. However, the grading of oligodendroglial tumors is still based purely on histology. Anaplastic (high-grade) oligodendroglioma accounts for about $30 \%$ of cases according to the 2007 WHO classification and predicts a much poorer outcome compared to nonanaplastic tumors $[14,15]$. However, interobserver variability in the grading of oligodendroglioma has been reported to be as high as $20 \%$ [1619].

Despite recent progress in understanding the molecular pathogenesis and disease course of glioma, research in pODG is scarce. A comprehensive review of the literature identified 15 studies that investigate the demographics and risk factors of pODG [20-34], and only 7 that examine factors related to prognosis [21, 22, 25, 28, 29, 32, 34]. Among these studies, only 3 analyze cases from more than 1 institution $[21,25,26]$ and none includes more than 50 patients. All prior studies identified relied on the 2007 WHO criteria for diagnosis.

These studies find that rates of 5 -year overall survival (OS) in pODG range from 65 to $94 \%$ [22, 25, 29]. Age of diagnosis greater than 12 years and tumor location outside of the cerebral cortex have both been linked to poor outcome $[22,25,29]$. Furthermore, a preponderance of data shows that $1 \mathrm{p} / 19 \mathrm{q}$ chromosomal deletion and IDH1 mutation - markers of good prognosis in adults - occur much less frequently in pediatric patients compared to adults $[21,23,24,26]$, among tumors classified histologically as oligodendroglioma. As mentioned previously though, earlier studies are limited by small population

Outcomes in Pediatric

Oligodendroglioma sizes and relatively short length of follow-up, making it difficult to carefully analyze long-term prognostic factors in $\mathrm{pODG}$.

In our study, we evaluated 346 patients diagnosed with pODG according to 2007 WHO criteria in the Surveillance, Epidemiology, and End Results (SEER) database from the National Cancer Institute - examining demographics, treatment patterns, prognostic factors, and outcomes as compared with oligodendroglioma in adults. To our knowledge, this is the largest retrospective study of outcomes in oligodendroglioma among patients who are under 18 years old by a considerable degree.

\section{Methods}

Data were accessed from the November 2016 submission of the SEER-18 registry, which includes cases diagnosed between 1973 and 2013. A diagnosis of oligodendroglioma was defined by International Classification of Diseases for Oncology, Third Edition (ICD-O-3) morphology codes 9450/3, 9451/3, and $9460 / 3$. These codes rely on 2007 WHO diagnostic criteria and are based only on tumor histology. Data on genetic biomarkers are not available from the SEER database. Patients diagnosed before the age of 18 were included in the pediatric population, and all others were considered adults. Any patient for whom oligodendroglioma was not the first primary malignancy recorded in the SEER database was excluded from analysis. Analysis was focused on the following demographic, tumor, and treatment factors: age at diagnosis, gender, race, tumor size, tumor location, tumor grade, use of radiotherapy, and extent of surgical resection. Chemotherapy data were not available from the SEER database.

\section{Statistical Analysis}

Univariate comparison of demographic, tumor, and treatment factors among pediatric and adult patients was made using the Pearson $\chi^{2}$ test. Overall survival was calculated as the number of months from diagnosis to date of death, and patient follow-up was censored at 240 months. The median length of follow-up was 115 months. Separate Kaplan-Meier curves were generated for both patient populations assessing the effect on overall survival of gender, race, tumor size, tumor location, tumor grade, use of radiotherapy, and extent of surgical resection. Survival comparisons were made using the log-rank test. A multivariate Cox proportional hazards model was built using each of the data points listed above as covariates to generate hazard ratios and corresponding $95 \%$ confidence intervals. Findings were considered statistically significant at $\mathrm{p}$ values $<0.05$. All analyses were performed using IBM SPSS Statistics for Macintosh, version 22 (IBM Corp., Armonk, NY, USA).
Pediatr Neurosurg 2018;53:24-35 DOI: $10.1159 / 000481458$ 
Table 1. Comparison of demographics, tumor factors, and treatment information in children and adults

\begin{tabular}{|c|c|c|c|c|c|}
\hline & Pediatric, $n$ & Adult, $n$ & $p$ value & Pediatric, \% & Adult, $\%$ \\
\hline \multicolumn{6}{|l|}{ Gender } \\
\hline Male & 192 & 3,256 & \multirow[t]{3}{*}{0.687} & 55 & 57 \\
\hline Female & 154 & 2,497 & & 45 & 43 \\
\hline Unknown & 0 & 0 & & 0 & 0 \\
\hline \multicolumn{6}{|l|}{ Race } \\
\hline White & 287 & 5,067 & \multirow[t]{5}{*}{$<0.0001$} & 83 & 89 \\
\hline Black & 40 & 230 & & 12 & 4 \\
\hline Native American & 6 & 49 & & 2 & 1 \\
\hline Asian & 11 & 361 & & 3 & 6 \\
\hline Unknown & 2 & 46 & & 1 & 1 \\
\hline \multicolumn{6}{|l|}{ Tumor location } \\
\hline Frontal lobe & 73 & 2,923 & \multirow[t]{6}{*}{$<0.0001$} & 22 & 53 \\
\hline Temporal lobe & 107 & 982 & & 32 & 18 \\
\hline Parietal lobe & 48 & 601 & & 14 & 11 \\
\hline Extracortical & 62 & 253 & & 19 & 5 \\
\hline Overlapping/other & 42 & 774 & & 13 & 14 \\
\hline Unknown & 14 & 220 & & 4 & 4 \\
\hline \multicolumn{6}{|l|}{ Tumor size } \\
\hline$>3 \mathrm{~cm}$ & 81 & 2,474 & \multirow[t]{3}{*}{$<0.0001$} & 45 & 76 \\
\hline$\leq 3 \mathrm{~cm}$ & 99 & 767 & & 55 & 24 \\
\hline Unknown & 166 & 2,512 & & 48 & 44 \\
\hline \multicolumn{6}{|l|}{ Tumor grade } \\
\hline Anaplastic & 53 & 1,950 & \multirow[t]{3}{*}{$<0.0001$} & 28 & 46 \\
\hline Nonanaplastic & 138 & 2,325 & & 72 & 54 \\
\hline Unknown & 155 & 1,478 & & 45 & 26 \\
\hline \multicolumn{6}{|l|}{ Radiotherapy } \\
\hline Yes & 74 & 2,840 & \multirow[t]{3}{*}{$<0.0001$} & 21 & 51 \\
\hline No & 272 & 2,739 & & 79 & 49 \\
\hline Unknown & 0 & 174 & & 0 & 3 \\
\hline \multicolumn{6}{|l|}{ Surgery } \\
\hline Gross total resection & 149 & 1,880 & \multirow[t]{4}{*}{$<0.0001$} & 50 & 36 \\
\hline Partial resection & 113 & 2,293 & & 38 & 44 \\
\hline No surgery & 34 & 1,077 & & 11 & 21 \\
\hline Unknown & 50 & 503 & & 14 & 9 \\
\hline
\end{tabular}

Unknown percentages are expressed as a fraction of all cases (known and unknown). All other percentages are expressed as a fraction of all cases whose value is known. $p$ values are calculated among known cases only.

\section{Results}

\section{Demographics, Tumor Factors, and Treatment}

\section{Patterns}

In total, 346 pediatric patients and 5,753 adult patients were included in the analysis. More than half of these patients were diagnosed in 2001 or later. The median age of diagnosis among pediatric and adult patients was 11 and 44 years, respectively. The median follow-up time was 115 months. The demographic, tumor, and treatment information for both pediatric and adult cohorts is given in Table 1. In both cohorts, the incidence was greater in males than in females, with $55 \%$ of pediatric cases occurring in males and $57 \%$ of adult cases. The racial composition of the 2 cohorts differed significantly. In the pediatric population, disease was more common in patients of African descent (12 vs. $4 \%$ ) and less common in patients of Asian descent (3 vs. 6\%) as compared with adults. There were also significant differences in tumor location. In adults, a majority of oligodendroglioma arose in the frontal lobe $(53 \%)$. In children, only $22 \%$ of tumors occurred in the frontal lobe, whereas the temporal lobe (32 vs. $18 \%$ in adults) and extracortical regions of the cerebrum (19 vs. $5 \%$ in adults) were much more common locations of 


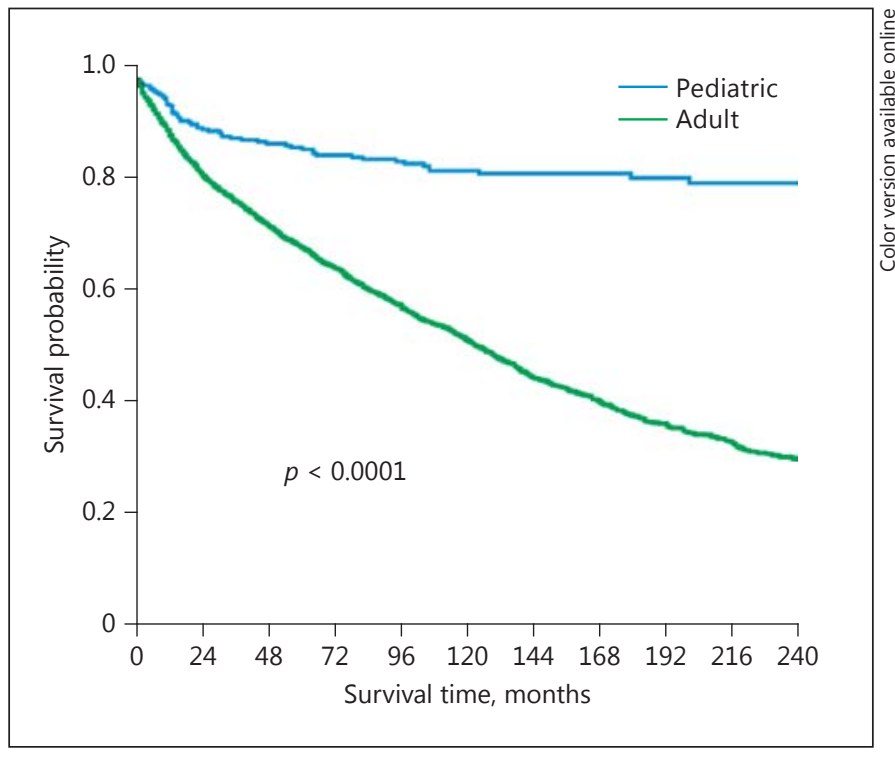

Fig. 1. Kaplan-Meier curve showing the 20-year overall survival of pediatric and adult patients with oligodendroglioma.

tumor origin. Among extracortical tumors in pediatric patients, 31 occurred in the diencephalon or basal ganglia, 13 in the ventricles, 12 in the cerebellum, and 6 in the brainstem. Tumor locations represented by the "Other" category included the occipital lobe, spinal cord, cauda equina, olfactory nerve, and pineal gland. Together, these account for only $4.3 \%$ of pediatric cases and $1.7 \%$ of adult cases. All brain regions were specified by ICD-O-3 topography codes in the SEER database.

For both the size of tumor at diagnosis and tumor grade, almost half of all cases in both cohorts were unspecified in the SEER database. Among cases for which these data were available, pODG was associated with smaller and lower-grade tumors to a significant degree. Only $45 \%$ of pediatric tumors were $>3 \mathrm{~cm}$ in size at the time of diagnosis compared with $76 \%$ of adult tumors. Similarly, just $28 \%$ of pediatric tumors were designated anaplastic (high-grade) oligodendroglioma at diagnosis compared with $46 \%$ of adult tumors, according to 2007 WHO criteria.

Patterns of treatment were also found to differ significantly among pediatric and adult patients. Pediatric patients were much less likely to receive radiotherapy than adults ( 21 vs. $51 \%$ ) but more likely to undergo gross total resection of their tumor - as opposed to partial tumor resection or no surgery - compared with adults (50 vs. $36 \%)$.

Outcomes in Pediatric

Oligodendroglioma

\section{OS and Prognostic Factors}

OS was significantly more favorable in pODG as compared with adult disease (Fig. 1). The mean OS in the pediatric cohort was 199.6 months with a standard error of 4.7 months. The 5 -year survival rate was $85 \%$, and the 10 -year survival rate was $81 \%$. For adults, the mean OS was $129.5 \pm 1.4$ months, the 5 -year survival rate was $67 \%$, and the 10 -year survival rate was $51 \%$.

Neither gender nor race were associated with survival in the pediatric cohort (Fig. 2a, c), whereas women showed a slightly better prognosis than men in adult disease (Fig. 2b). Prognosis with respect to tumor location differed among the 2 cohorts (Fig. 2e, f). In adults, frontal lobe tumors showed the best prognosis among all CNS regions, with a significantly better 10 -year survival (58\%) compared to tumors of the parietal and temporal lobes (both 47\%). In the pediatric cohort, tumors of the temporal lobe showed the best prognosis among all CNS regions, although there was little survival difference among regions of the cerebral cortex in pODG, and the relatively small number of events in each group makes careful analysis difficult. In both cohorts, tumors of the extracortical cerebrum showed the poorest survival among all CNS regions in the first 5 years after diagnosis.

Prognosis with respect to tumor size differed among the 2 cohorts as well (Fig. $2 \mathrm{~g}, \mathrm{~h}$ ). Among pediatric patients, tumor size was a strong prognostic factor. Patients with tumors $>3 \mathrm{~cm}$ fared significantly worse and were especially prone to death within 5 years of diagnosis. In contrast, in adult patients tumor size showed no association with survival within the first 5 years, but did influence survival at 10 and 20 years after diagnosis. While high tumor grade predicted poor prognosis among all patients, high grade was another particularly strong negative prognostic factor in children (Fig. 2i, j). Rates of 10year OS in pediatric patients with anaplastic (high-grade) oligodendroglioma were just $42 \%$ compared with $93 \%$ for nonanaplastic tumors. In adults, 10-year OS was 38 and $45 \%$ for anaplastic and nonanaplastic oligodendroglioma, respectively.

Predictably, surgical and radiological interventions affected survival in both cohorts as well (Fig. 2k-n). Radiotherapy was associated with poor survival in both cohorts - almost certainly a product of the strong correlation between high tumor grade and the decision to treat with radiotherapy (data not shown). In both cohorts, greater extent of tumor resection showed a robust relationship with improved prognosis.

Comparison of OS was also made among equivalent groups of pediatric and adult cases. Among cases matched

Pediatr Neurosurg 2018;53:24-35

DOI: $10.1159 / 000481458$ 


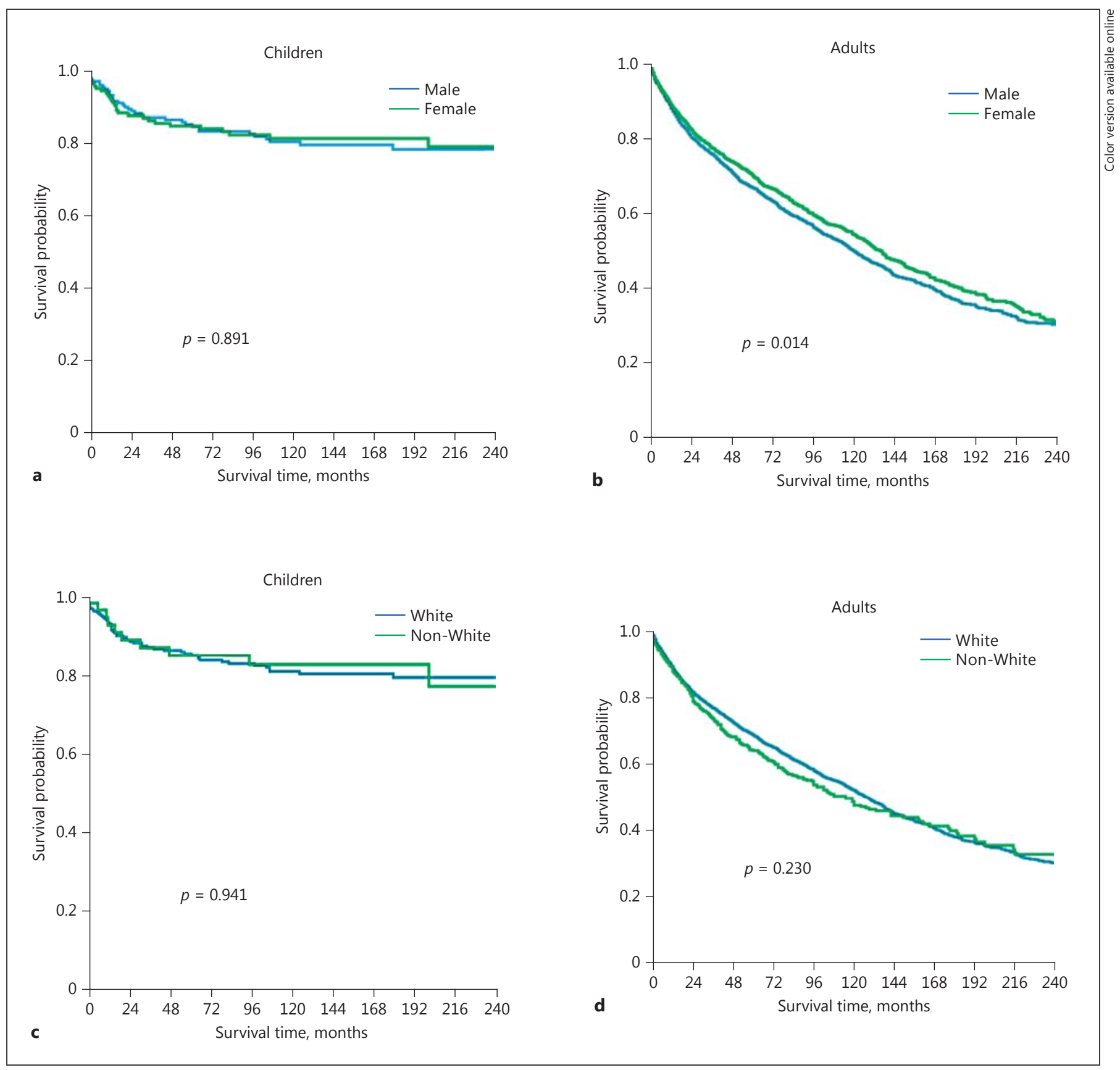

Fig. 2. Kaplan-Meier curves comparing the influence on 20 -year overall survival in pediatric and adult oligodendroglioma of gender $(\mathbf{a}, \mathbf{b})$, race $(\mathbf{c}, \mathbf{d})$, tumor location within the brain $(\mathbf{e}, \mathbf{f})$, tumor size at diagnosis $(\mathbf{g}, \mathbf{h})$, tumor grade at diagnosis $(\mathbf{i}, \mathbf{j})$, use of radiotherapy $(\mathbf{k}, \mathbf{I})$, and extent of surgical resection $(\mathbf{m}, \mathbf{n})$.

(Figure continued on next pages.)

for gender, race, tumor size, tumor location, use of radiotherapy, and type of surgery, survival was significantly longer for pediatric patients in all categories. However, this was not true for cases matched by tumor grade. No survival difference was observed between pediatric high- grade and adult high-grade tumors (Fig. 3a). Among lowgrade tumors though, pediatric patients demonstrated better outcomes than adults (Fig. 3b).

Previous studies of pODG have reported differences in both molecular features and outcomes between tumors of 


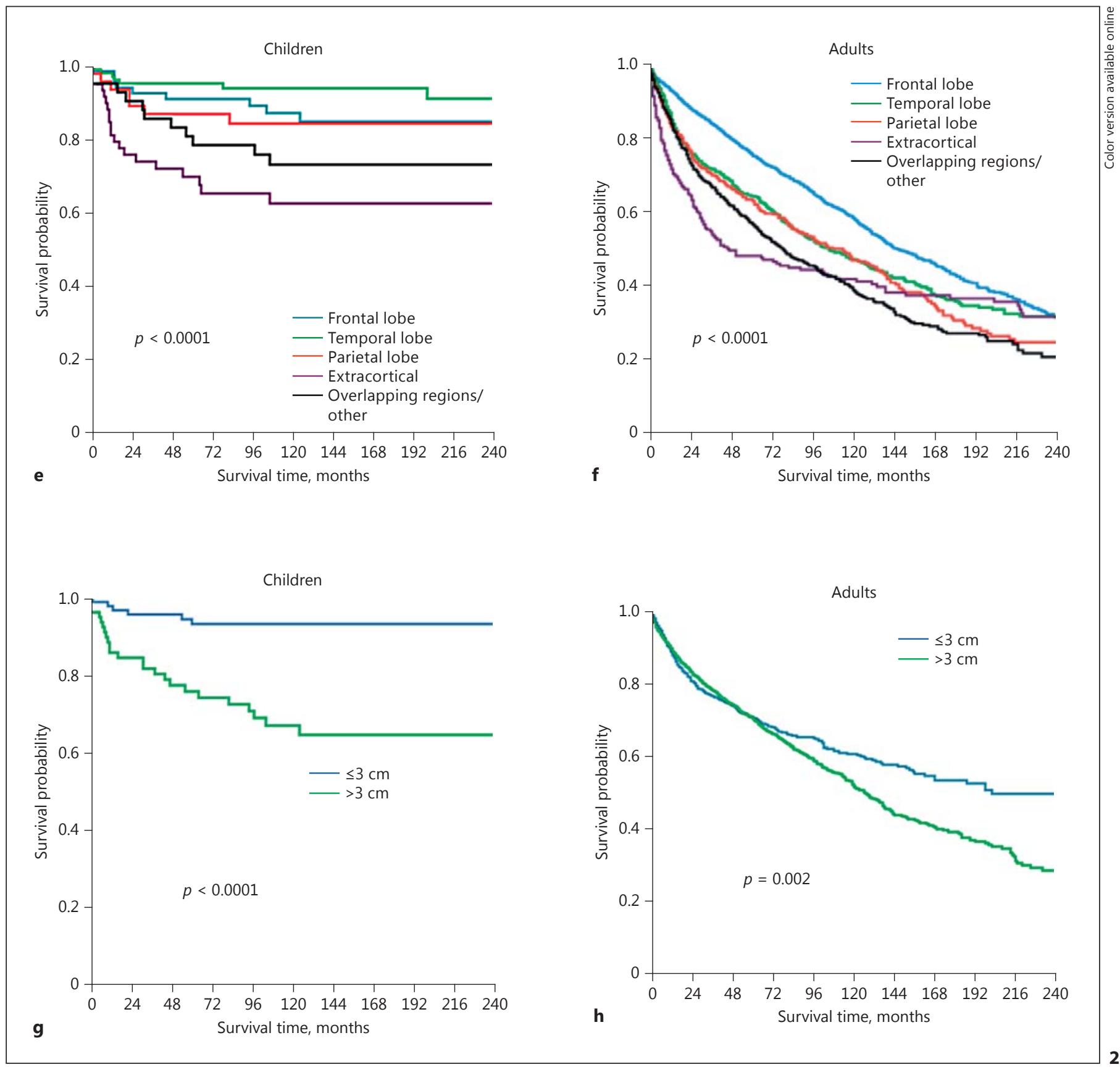

early childhood and tumors of adolescence [22, 25, 29]. Based on these reports, we examined the relationship between age of diagnosis, prevalence, and survival within the pediatric cohort (Fig. 4). While prevalence of oligodendroglioma does begin to increase near the age of puberty onset (Fig. $4 \mathrm{~b}$ ), we found no difference in OS between young children and adolescents (Fig. 4a).

Outcomes in Pediatric

Oligodendroglioma
A multivariate Cox proportional hazards model of OS for the pediatric and adult cohorts is presented in Table 2 . On multivariate analysis, radiotherapy, extent of surgical resection, and tumor grade all demonstrated an independent association with survival in both cohorts. In adults only, tumor location was also an independent prognostic factor - all tumor locations showed diminished survival compared to the frontal lobe. No independent prognostic 


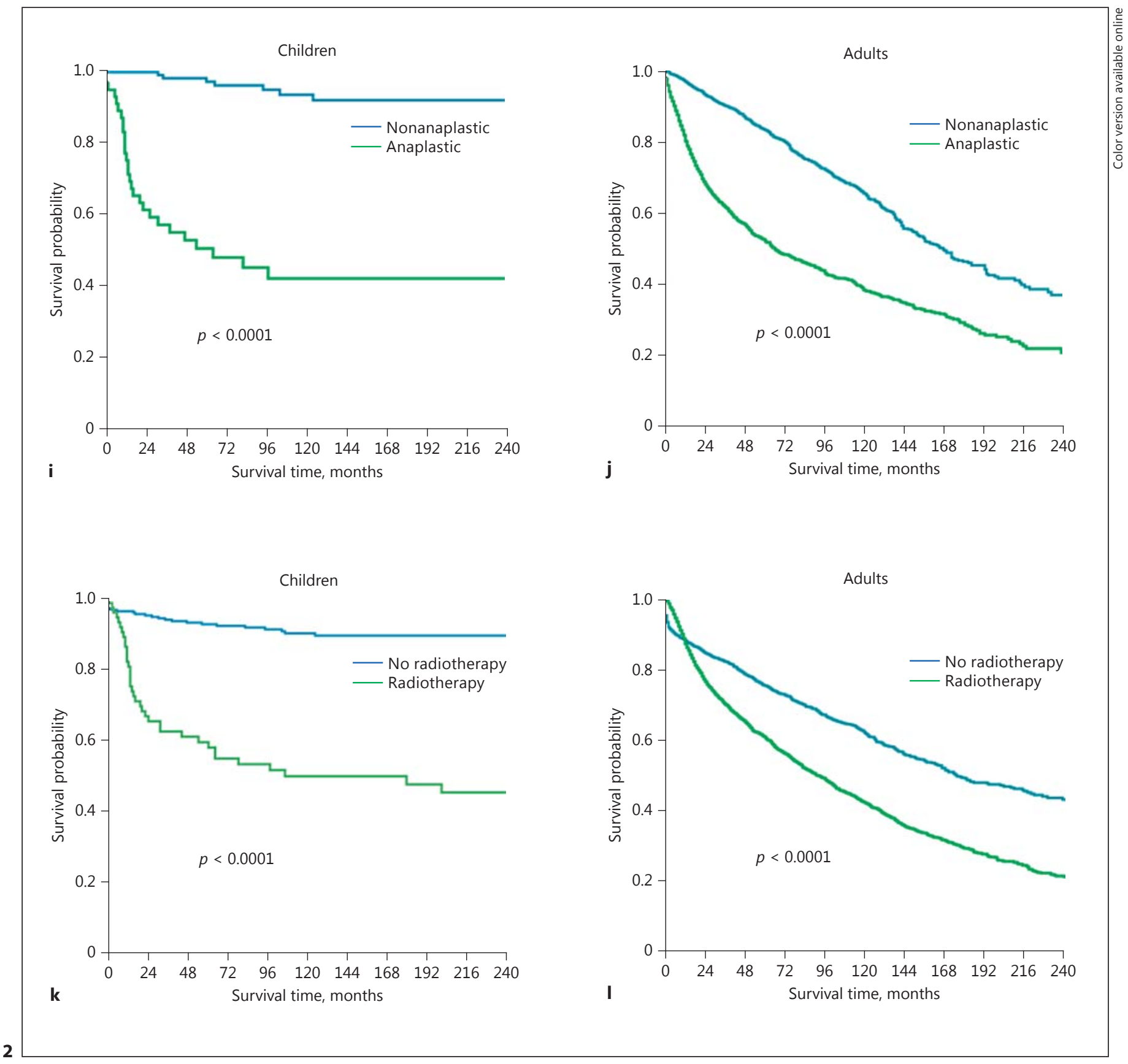

influence of tumor location was observed in children. Conversely, tumor size was a very strong independent prognostic factor in children $(\mathrm{HR}=3.9 ; \mathrm{CI}=1.3-12.2)$ but showed no prognostic influence in adults $(\mathrm{HR}=1.0 ; \mathrm{CI}=$ $0.8-1.2)$. The Cox proportional hazards model constructed for the pediatric cohort was somewhat underpowered, including only 119 cases and 24 events compared to the adult model which included 2,513 cases and 914 events.
Thus, that tumor size proved such a strong independent prognostic factor in children, while no independent effect could be detected in the more highly powered adult cohort, heavily suggests that the true underlying influence of tumor size on prognosis is considerably greater in children than in adults. Tumor grade was the single strongest prognostic factor in pODG in both univariate and multivariate analysis $(\mathrm{HR}=6.6 ; \mathrm{CI}=1.9-23.0)$. 

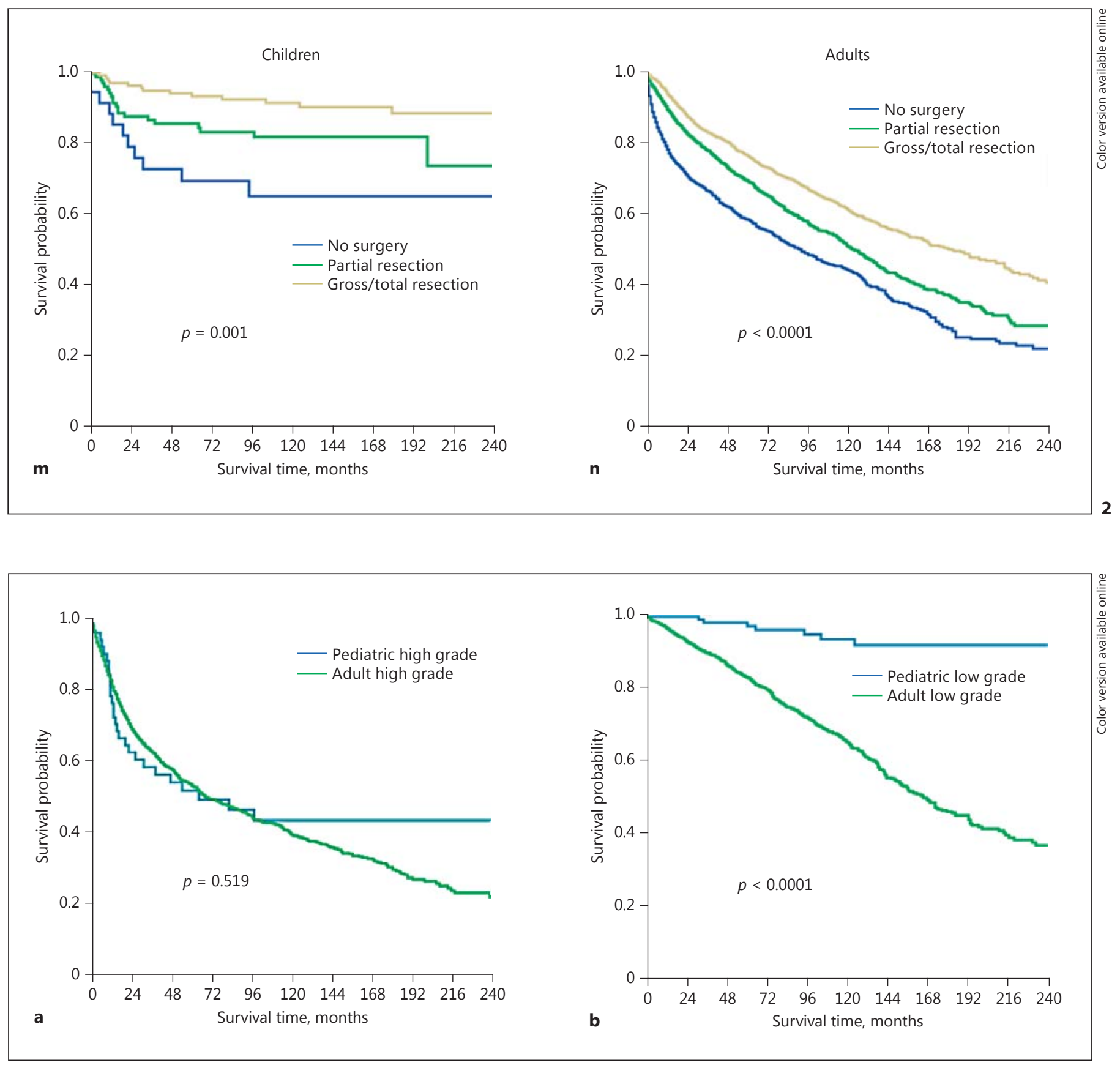

Fig. 3. Kaplan-Meier curves comparing overall survival between pediatric and adult cases matched for high tumor grade (a) and low tumor grade (b).

\section{Discussion}

Our study of 346 cases represents the largest retrospective study of oligodendroglioma in the under 18-year-old population in the literature to date. A comprehensive review of the literature identified only 7 previous studies that analyzed prognostic factors in pODG $[21,22,25,28$, $29,32,34]$, of which only 3 include cases from more than 1 institution $[21,25,26]$. The largest of these studies by Rodriguez et al. [21] reports only 50 cases.

In our interrogation of the SEER database, we found that OS in PODG is significantly better than that of adult 


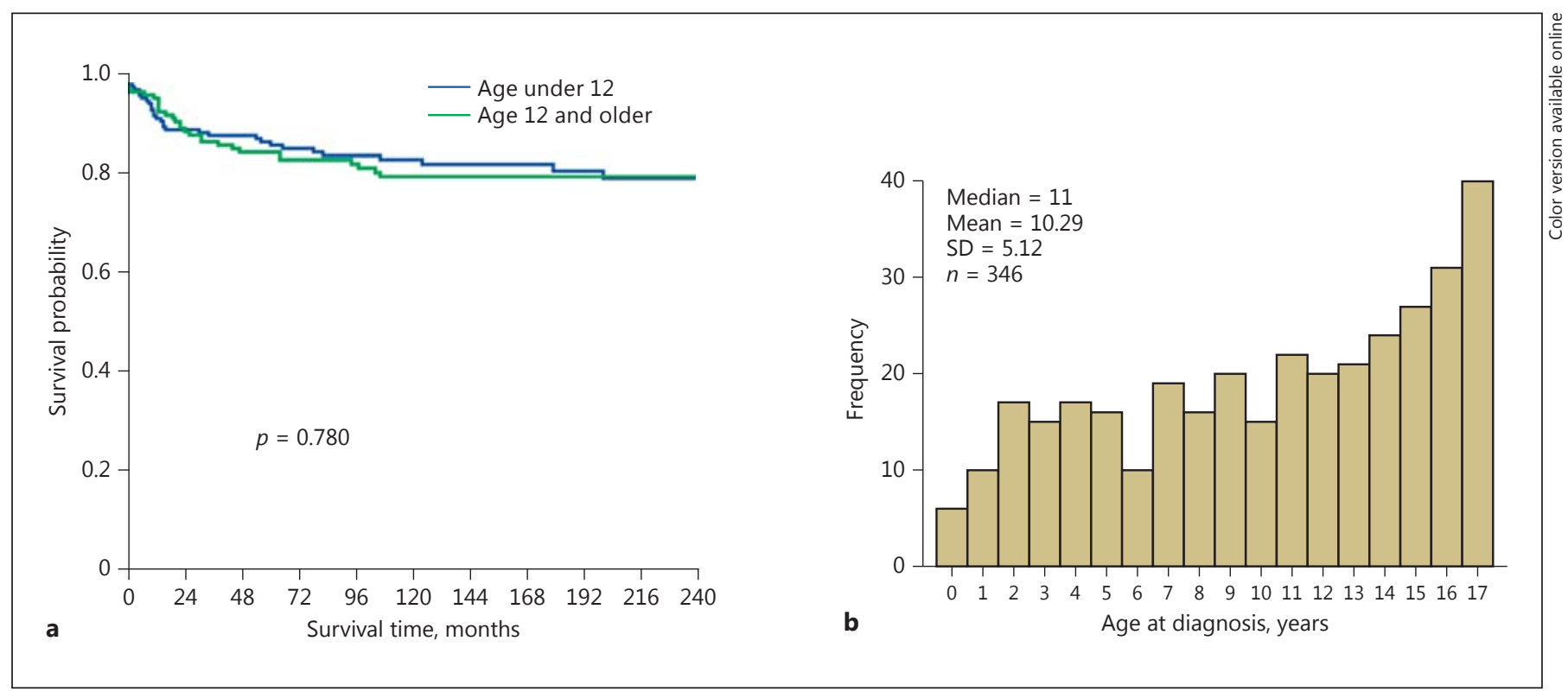

Fig. 4. The significance of age at diagnosis within the pediatric cohort. a Kaplan-Meier curve showing the effect of age on 20-year overall survival. b Histogram showing the incidence of pediatric oligodendroglioma by age.

oligodendroglioma, with a 5 -year OS of $85 \%$. These results are roughly in agreement with results of previous studies. Rodriguez et al. [21] report a 5-year OS of $80 \%$, Creach et al. [22] report 94\%, Peters et al. [25] report 84\%, and Razack et al. [29] report 65\%. This compares with a lower 5 -year OS in adult oligodendroglioma of $67 \%$ identified in our study.

It would seem that a large portion of the improved outcome in pODG stems from the relatively greater fraction of pediatric tumors presenting at a lower grade than adult tumors - as grade was the strongest of all prognostic factors in both cohorts. We observed that $72 \%$ of pediatric cases were of low grade (grade II) according to 2007 WHO criteria as compared with $54 \%$ of adult cases. This result is supported in the pediatric neuro-oncology literature as well. Creach et al. [22] identified $80 \%$ of 37 pODG cases as low grade while Rodriguez et al. [21] identified $76 \%$ of 50 pODG cases as low grade. It is also important to highlight our finding that the improved survival seen in children compared with adults with oligodendroglioma applies only to low-grade tumors. No survival difference was seen between adults and children with highgrade disease.

Another key clinical difference observed between pODG and adult oligodendroglioma was tumor location within the CNS. We found that $19 \%$ of pODG tu- mors presented in extracortical regions of the cerebrum that include the cerebellum, brainstem, diencephalon and ventricles, whereas only $5 \%$ of adult tumors were found in these regions. Extracortical tumors were associated with the worst prognosis of any region in the CNS in pODG. Rodriguez et al. [21] also note the tendency of pODG to develop in extracortical regions rarely associated with adult disease. Peters et al. [25] further report 9 of 32 cases analyzed appearing in these extracortical regions and note that such cases were associated with a significantly worse survival. Tumor location within the cerebral cortex also differed among pediatric and adult cohorts. In adults, we found that 55\% of all oligodendrogliomas arose in the frontal lobe. However, in children we found that the temporal lobe was a more common location of disease than the frontal lobe. Interestingly, both Peters et al. [25] and Razack et al. [29] also find that the temporal lobe is a more common location of pODG than the frontal lobe, whereas Rodriguez et al. [21] find the opposite. While pediatric tumors do tend toward locations in the brain associated with poorer survival, overall survival in pODG is still much more favorable than in adult disease. This is likely because the tendency of pODG toward lower-grade tumors (noted above) dominates the effect on survival of the tendency toward less favorable tumor location - as tumor grade 
Table 2. Multivariate analysis of 20 -year OS by Cox proportional hazards model

\begin{tabular}{|c|c|c|c|c|}
\hline & \multicolumn{2}{|l|}{ Pediatric cohort } & \multicolumn{2}{|l|}{ Adult cohort } \\
\hline & Hazard ratio $(95 \% \mathrm{CI})$ & $p$ value & Hazard ratio $(95 \% \mathrm{CI})$ & $p$ value \\
\hline \multicolumn{5}{|l|}{ Gender } \\
\hline Male & 1.00 & - & 1.00 & - \\
\hline Female & $0.61(0.23-1.60)$ & 0.31 & $0.89(0.77-1.00)$ & 0.06 \\
\hline \multicolumn{5}{|l|}{ Race } \\
\hline White & 1.00 & - & 1.00 & - \\
\hline Non-White & $1.90(0.55-6.50)$ & 0.32 & $1.04(0.85-1.27)$ & 0.71 \\
\hline \multicolumn{5}{|l|}{ Tumor size } \\
\hline$\leq 3 \mathrm{~cm}$ & 1.00 & - & 1.00 & - \\
\hline$>3 \mathrm{~cm}$ & $3.93(1.26-12.21)$ & 0.02 & $1.00(0.84-1.17)$ & 0.96 \\
\hline \multicolumn{5}{|l|}{ Tumor grade } \\
\hline Nonanaplastic & 1.00 & - & 1.00 & \\
\hline Anaplastic & $6.58(1.89-22.97)$ & 0.003 & $2.66(2.30-3.08)$ & $<0.0001$ \\
\hline \multicolumn{5}{|l|}{ Tumor location } \\
\hline Frontal lobe & 1.00 & - & 1.00 & - \\
\hline Temporal lobe & $0.29(0.03-2.7)$ & 0.27 & $2.15(1.80-2.57)$ & $<0.0001$ \\
\hline Parietal lobe & $0.54(0.09-3.13)$ & 0.49 & $1.41(1.15-1.74)$ & 0.00 \\
\hline Extracortical & $1.65(0.45-6.05)$ & 0.45 & $2.90(2.15-3.91)$ & $<0.0001$ \\
\hline Multiple sites/other & $1.37(0.31-6.13)$ & 0.68 & $1.78(1.48-2.14)$ & $<0.0001$ \\
\hline \multicolumn{5}{|l|}{ Radiotherapy } \\
\hline No & 1.00 & - & 1.00 & - \\
\hline Yes & $2.90(0.84-10.36)$ & 0.09 & $1.33(1.14-1.54)$ & $<0.001$ \\
\hline \multicolumn{5}{|l|}{ Surgery } \\
\hline No surgery & 1.00 & - & 1.00 & - \\
\hline Partial resection & $0.22(0.06-0.84)$ & 0.03 & $0.78(0.65-0.93)$ & 0.01 \\
\hline Gross total resection & $0.52(0.14-2.00)$ & 0.34 & $0.64(0.52-0.77)$ & $<0.0001$ \\
\hline
\end{tabular}

is a much stronger prognostic factor than tumor location.

Many previous studies of histologically defined pODG have found that the genetic biomarkers most typical of oligodendroglioma, namely $1 \mathrm{p} / 19 \mathrm{q}$ chromosomal codeletion and IDH1 mutation, occur less frequently in pediatric tumors $[21,23,24,26]$. Data also suggest that the absence of these markers is particularly pronounced in the youngest patients of the pediatric cohort $[21,23,26]$. Based on these findings, Rodriguez et al. [21] speculate that so-called childhood-type oligodendroglioma predominates in patients younger than 15 years and that these tumors progress through a distinct molecular pathway independently of the IDH1 and 1p/19q genetic biomarkers. Along these lines, 3 studies - Rodriguez et al. [21], Creach et al. [22], and Razack et al. [29] - analyzed age-specific survival within the pediatric cohort. Rodriguez et al. report no association between age and survival while Creach et al. report that children under 12 years

Outcomes in Pediatric

Oligodendroglioma show worse prognosis, and Razack et al. also identified the same trend, albeit without statistical significance. Our data indicate no age-specific prognostic value within the pediatric cohort. Careful analysis of pathological and cytogenetic features in a large cohort - such as the one reported on in this study - is needed to elucidate the molecular basis of pODG and the effect this may have on treatment or prognosis.

There are a few important limitations of this study to note. Because the SEER database does not include data regarding genetic biomarkers, the classification of a tumor as oligodendroglioma in this study was made purely on histological phenotype, according to the 2007 WHO guidelines. According to the most recent guidelines released in 2016, demonstrations of IDH mutation and $1 \mathrm{p} / 19 \mathrm{q}$ codeletion are both required for the diagnosis of oligodendroglioma, taking precedence over histology. Thus, many tumors that demonstrate the classic features of oligodendroglioma under traditional

Pediatr Neurosurg 2018;53:24-35 DOI: $10.1159 / 000481458$ 
histology - and would be characterized as such in this study - are no longer considered to be oligodendroglioma under the current criteria. Moreover, there are some data to suggest that oligodendroglioma-like tumors that lack the appropriate genetic phenotype are particularly common in the pediatric population $[13,21,23,26]$. Such tumors may presently be best characterized as diffuse astrocytoma or histological mimics of pODG such as pilocytic astrocytoma or dysembryoplastic neuroepithelial tumor. This recent shift in the classification of pODG may in the future limit the clinical utility of this study. It is also important to note that no data pertaining to chemotherapy for primary tumors of the CNS are available in the SEER database. Although there exists no clear standard chemotherapeutic regimen in oligodendroglioma, these tumors are generally found to be much more sensitive to chemotherapy than most other primary brain tumors. Chemotherapy is thus an important part of oligodendroglioma treatment and potentially drives some differences in outcomes among olidodendroglioma cases.

\section{Conclusion}

In summary, the present study represents the largest retrospective analysis of demographics, outcomes, and prognostic factors in oligodendroglioma in patients under 18 years old to date. We find that pODG differs from adult oligodendroglioma along a number of demographic and tumor factors such as race, tumor size, tumor location, and tumor grade. Furthermore, key prognostic factors including tumor size and tumor location are found to influence survival in pODG differently from adult disease.

\section{Statement of Ethics}

Since all patient information was de-identified prior to access by the authors of this study, no IRB approval was required.

\section{Disclosure Statement}

The authors have no conflicts of interest to disclose.

\section{References}

1 Ostrom QT, Gittleman H, Liao P, Rouse C, Chen Y, Dowling J, Wolinsky Y, Kruchko C, Barnholtz-Sloan J: CBTRUS statistical report: primary brain and central nervous system tumors diagnosed in the United States in 20072011. Neuro Oncol 2014;16:1-63.

2 Qaddoumi I, Sultan I, Gajjar A: Outcome and prognostic features in pediatric gliomas: a review of 6,212 cases from the Surveillance, Epidemiology, and End Results (SEER) database. Cancer 2009;115:5761-5770.

3 Davis FG, Freels S, Grutch J, Barlas S, Brem S: Survival rates in patients with primary malignant brain tumors stratified by patient age and tumor histological type: an analysis based on Surveillance, Epidemiology, and End Results (SEER) data, 1973-1991. J Neurosurg 1998;88:1-10.

4 Van den Bent MJ, Bromberg JE, Buckner J: Low-grade and anaplastic oligodendroglioma. Handb Clin Neurol 2016;134:361-380.

5 Minniti G, Arcella A, Scaringi C, Lanzetta G, Di Stefano D, Scarpino S, Pace A, Giangaspero F, Osti MF, Enrici RM: Chemoradiation for anaplastic oligodendrogliomas: clinical outcomes and prognostic value of molecular markers. J Neurooncol 2014;116:275-282.

6 Wesseling P, van den Bent M, Perry A: Oligodendroglioma: pathology, molecular mechanisms and markers. Acta Neuropathol 2015; 129:809-827.
7 Louis DN, Ohgaki H, Wiestler OD, Cavenee WK, Burger PC, Jouvet A, Scheithauer BW, Kleihues P: The 2007 WHO classification of tumours of the central nervous system. Acta Neuropathol 2007;114:97-109.

8 Smith JS, Alderete B, Minn Y, Borell JT, Perry A, Mohapatra G, Hosek S, Kimmel D, O'Fallon J, Yates A, Feuerstein BG, Burger PC, Scheithauer BW, Jenkins RB: Localization of common deletion regions on $1 p$ and $19 \mathrm{q}$ in human gliomas and their association with histological subtype. Oncogene 1999;18: 4144-4152.

9 Reifenberger J, Reifenberger G, Liu L, James CD, Wechsler W, Collins VP: Molecular genetic analysis of oligodendroglial tumors shows preferential allelic deletions on $19 \mathrm{q}$ and 1p. Am J Pathol 1994;145:1175-1190.

10 Yan H, Parsons DW, Jin G, McLendon R, Rasheed BA, Yuan W, Kos I, Batinic-Haberle I, Jones S, Riggins GJ, Friedman H, Friedman A, Reardon D, Herndon J, Kinzler KW, Velculescu VE, Vogelstein B, Bigner DD: IDH1 and IDH2 mutations in gliomas. $\mathrm{N}$ Engl J Med 2009;360:765-773.
11 Smith JS, Perry A, Borell TJ, Lee HK, O’Fallon J, Hosek SM, Kimmel D, Yates A, Burger PC, Scheithauer BW, Jenkins RB: Alterations of chromosome arms $1 p$ and $19 q$ as predictors of survival in oligodendroglioma, astrocytomas, and mixed oligastrocytomas. J Clin Oncol 2000;18:636-645.

12 Cairncross JG, Ueki K, Zlatescu MC, Lisle DK, Finkelstein DM, Hammond RR, Silver JS, Stark PC, Macdonald DR, Ino Y, Ramsay DA, Louis DN: Specific genetic predictors of chemotherapeutic response and survival in patients with anaplastic oligodendrogliomas. J Natl Cancer Inst 1998;90:1473-1479.

13 Louis DN, Perry A, Reifenberger G, von Deimling A, Figarella-Branger D, Cavenee WK, Ohgaki H, Wiestler OD, Kleihues P, Ellison DW: The 2016 World Health Organization Classification of Tumors of the Central Nervous System: a summary. Acta Neuropathol 2016;131:803-820.

14 Simonetti G, Gaviani P, Botturi A, Innocenti A, Lamperti E, Silvani A: Clinical management of grade III oligodendroglioma. Cancer Manag Res 2015;7:213-223.

15 Shaw EG, Scheithauer BW, O'Fallon JR: Supratentorial gliomas: a comparative study by grade and histologic type. J Neurooncol 1997; 31:273-278. 
16 Giannini C, Scheithauer BW, Weaver AL, Burger PC, Kros JM, Mork S, Graeber MB, Bauserman S, Buckner JC, Burton J, Riepe R, Tazelaar HD, Nascimento AG, Crotty T, Keeney GL, Pernicone P, Altermatt H: Oligodendrogliomas: reproducibility and prognostic value of histologic diagnosis and grading. J Neuropathol Exp Neurol 2001;60:248-262.

17 Kros JM, Gorlia T, Kouwenhoven MC, Zheng PP, Collins VP, Figarella-Branger D, Giangaspero F, Giannini C, Mokhtari K, Mørk SJ, Paetau A, Reifenberger G, van den Bent MJ: Panel review of anaplastic oligodendroglioma from European Organization for Research and Treatment of Cancer Trial 26951: assessment of consensus in diagnosis, influence of $1 \mathrm{p} / 19 \mathrm{q}$ loss, and correlations with outcome. J Neuropathol Exp Neurol 2007;66:545-551.

18 Van den Bent MJ: Interobserver variation of the histopathological diagnosis in clinical trials on glioma: a clinician's perspective. Acta Neuropathol 2010;120:297-304.

19 Coons SW, Johnson PC, Scheithauer BW, Yates AJ, Pearl DK: Improving diagnostic accuracy and interobserver concordance in the classification and grading of primary gliomas. Cancer 1997;79:1381-1393.

20 Nauen D, Haley L, Lin MT, Perry A, Giannini C, Burger PC, Rodriguez FJ: Molecular analysis of pediatric oligodendrogliomas highlights genetic differences with adult counterparts and other pediatric gliomas. Brain Pathol 2016;26:206-214.

21 Rodriguez FJ, Tihan T, Lin D, McDonald W, Nigro J, Feuerstein B, Jackson S, Cohen K, Burger PC: Clinicopathologic features of pediatric oligodendrogliomas: a series of $50 \mathrm{pa}-$ tients. Am J Surg Pathol 2014;38:1058-1070.

22 Creach KM, Rubin JB, Leonard JR, Limbrick DD, Smyth MD, Dacey R, Rich KM, Dowling JL, Grubb RL Jr, Linette GP, King AA, Michalski JM, Park TS, Perry A, Simpson JR, Mansur DB: Oligodendrogliomas in children. J Neurooncol 2012;106:377-382.

23 Suri V, Jha P, Agarwal S, Pathak P, Sharma MC, Sharma V, Shukla S, Somasundaram K, Mahapatra AK, Kale SS, Sarkar C: Molecular profile of oligodendrogliomas in young patients. Neuro Oncol 2011;13:1099-1106.

24 Kreiger PA, Okada Y, Simon S, Rorke LB, Louis DN, Golden JA: Losses of chromosomes $1 \mathrm{p}$ and $19 \mathrm{q}$ are rare in pediatric oligodendrogliomas. Acta Neuropathol 2005;109: 387-392.

25 Peters O, Gnekow AK, Rating D, Wolff JE: Impact of location on outcome in children with low-grade oligodendroglioma. Pediatr Blood Cancer 2004;43:250-256.

26 Raghavan R, Balani J, Perry A, Margraf L, Vono MB, Cai DX, Wyatt RE, Rushing EJ, Bowers DC, Hynan LS, White CL: Pediatric oligodendrogliomas: a study of molecular al- terations on $1 p$ and $19 q$ using fluorescence in situ hybridization. J Neuropathol Exp Neurol 2003;62:530-537.

27 Myal Y, Del Bigio MR, Rhodes RH: Age-related differences in $1 p$ and $19 q$ deletions in oligodendrogliomas. BMC Clin Pathol 2003;3:6.

28 Bowers DC, Mulne AF, Weprin B, Bruce DA, Shapiro K, Margraf LR: Prognostic factors in children and adolescents with low-grade oligodendrogliomas. Pediatr Neurosurg 2002; 37:57-63.

29 Razack N, Baumgartner J, Bruner J: Pediatric oligodendrogliomas. Pediatr Neurosurg 1998;28:121-129.

30 Rizk T, Mottolèse C, Bouffet E, Jouvet A, Guyotat J, Bret P, Lapras C: Cerebral oligodendrogliomas in children: an analysis of 15 cases. Childs Nerv Syst 1996;12:527-529.

31 Tice H, Barnes PD, Goumnerova L, Scott RM, Tarbell NJ: Pediatric and adolescent oligodendroglioma. AJNR Am J Neuroradiol 1993; 14:1293-1300.

32 Wang KC, Chi JG, Cho BK: Oligodendroglioma in childhood. J Korean Med Sci 1993;8: 110-116.

33 Favier J, Pizzolato GP, Berney J: Oligodendroglial tumors in childhood. Childs Nerv Syst 1985;1:33-38.

34 Dohrmann GJ, Farewell JR, Flannery JT: Oligodendrogliomas in children. Surg Neurol 1978;10:21-25. 